\title{
Viscosity Solutions of Nonlinear Systems of Degenerated Elliptic Equations of Second Order
}

\author{
Liu Wei-an and Chen Hua
}

\begin{abstract}
In this paper, we discuss the viscosity solutions of Dirichlet problem for weakly coupled systems of fully nonlinear second order degenerated elliptic equations. We prove the existence, uniqueness and continuity of solutions by Perron's method combined with the technique of coupled solutions. Our results generalize those in [9] for the case of general quasi-monotonic systems.
\end{abstract}

Keywords: viscosity solutions, systems of partial differential equations, fully nonlinear degenerated elliptic equations, Perron's method, coupled solution

AMS subject classification: $35 \mathrm{~F} 30$

\section{Introduction}

In this paper we are concerned with the boundary problem of weakly coupled systems of fully nonlinear second order degenerated elliptic equations of the form

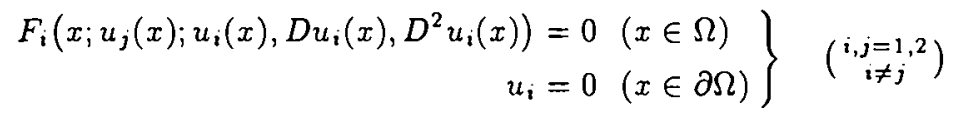

where

- $u=\left(u_{1}, u_{2}\right): \Omega \rightarrow \mathbb{R}^{2}$ is the unknown function

- $F=\left(F_{1}, F_{2}\right): \Omega \times \mathbb{R}^{2} \times \mathbb{R}^{n} \times \mathbf{S}(n) \rightarrow \mathbb{R}^{2}$ is a given locally bounded function

- $\mathrm{S}(n)$ is the set of real symmetric matrices of order $n$

- $D u_{i}=\left(\frac{\partial u_{i}}{\partial x_{k}}\right)(1 \leq k \leq n)$ is the gradient of $u_{i}$ with respect to $x$

- $D^{2} u_{i}=\left[\frac{\partial^{2} u_{i}}{\partial x_{k} \partial x_{l}}\right] \quad(1 \leq k, l \leq n)$ is the Hessian matrix of $u_{i}$

- $\Omega$ is a bounded open subset of $\mathbb{R}^{n}$.

The function $u_{i}$ is called the principal argument of $F_{i}$, while $u_{j}(j \neq i)$ is the coupled argument of $F_{i}$. Further, the function $F_{i}$ is said to be quasi-increasing (or decreasing) if

Both authors: Wuhan Univ., School of Math., Wuhan, Hubei, 430072 P.R. China liuweian@public.wh.hb.cn and.chenhua@whu.edu.cn

Supported by the National Natural Science Foundation of China and Hua-Cheng Funds.

ISSN 0232-2064 / \$ 2.50 (C) Heldermann Verlag Berlin 
it is increasing (or decreasing) with respect to the coupled argument $u_{j}$. Finally, system (1) is said to be

- quasi-increasing (or decreasing) if both functions $F_{1}$ and $F_{2}$ are quasi-increasing ( or decreasing);

- mixed quasi-monotonic if one of the functions $F_{1}$ or $\ddot{F}_{2}$ is quasi-increasing and the other function is quasi-decreasing (see $[19,20,23]$ ).

In 1983 Crandall and Lions [4] introduced the concept of viscosity solution, which is regarded as a kind of weak solution for first order fully nonlinear partial differential equations. They also established global uniqueness and existence of viscosity solutions, and showed that a classical solution is always a viscosity solution. Later, in P.L. Lions [17], the result has been extended to second order problems. Furthermore, from $[1,2$, $7,8,11]$, the theory of viscosity solutions has been completely developed and provided a powerful tool to deal with fully nonlinear partial differential equations. However, when we wish to study systems, even those with very special coupling structure, there are only a few results in this direction. In fact, if we regard a viscosity solution as a kind of comparison method, for scalar equations, the image of $F$ is in a total-ordered space which can be compared easily; but the image of $\left(F_{1}, \ldots, F_{m}\right)$ for a system is in a partial-ordered space in which trichotomy law is invalid. So it is more difficult to deal with systems, e.g. in order to use the maximum principle method, quasi-decreasing assumptions have been introduced in $[5,9,10,12,13]$. However, the comparison does not hold for sub-solutions and super-solutions in the classical definition of non-quasidecreasing systems.

In this paper, we introduce first a new definition of sub- and super-solutions for quasi-increasing systems or mixed-quasi-monotone systems making use of the technique of coupled solutions (the idea of couple solutions comes from $[6,20,23])$. Then, by Perron's method, existence and uniqueness of the viscosity solution for system (1) are proved, in which the system may be assumed to be quasi-increasing or mixed-quasimonotone. We also prove that the viscosity solution of system (1) is continuous even if $\left(F_{1}, F_{2}\right)$ is not continuous, thanks to the definition of sub-and super-solutions by locally bounded functions which comes from [9]. For non-quasi-monotone systems, we prove the same results by use of a fixed point theorem. Our results generalize those of Ishii and Koike [9] to the case of more general quasi-monotonic systems. Actually, in [9] they only discussed the case of quasi-decreasing systems (i.e. "quasi-monotone" in term of [9]).

For convenience, we discuss systems of two equations only. However, our results can be easily generalized to systems of arbitrary $m$ equations by introducing some appropriate notations used, for example, in [5, 9] and [19: Section 6.1].

In Section 2, we shall first give some results about scalar equations which will be used later in dealing with systems. In sections 3 and 4, we apply Perron's method, combined with technique of coupled solutions; to study quasi-increasing and mixed quasi-monotone systems, respectively. In Section 5, we prove comparison theorems for quasi-increasing and mixed quasi-monotone systems. In Section 6, we prove existence, uniqueness and continuity of viscosity solutions for non-quasi-monotonic systems by Banach fixed point theorem. Finally, in Section 7, some examples are presented to show that our results are non-trivial extensions of those in Ishii and Koike [9]. 


\section{Some results for scalar equations}

For a function $v: \bar{\Omega} \rightarrow \mathbb{R}$, its upper and lower envelopes are defined by

$$
\begin{aligned}
& v^{*}(x)=\limsup _{\varepsilon \rightarrow 0}\{v(y):|x-y|<\varepsilon \text { for } y \in \bar{\Omega}\} \\
& v_{*}(x)=\liminf _{\varepsilon \rightarrow 0}\{v(y):|x-y|<\varepsilon \text { for } y \in \bar{\Omega}\}
\end{aligned}
$$

respectively. It is clear from the definition that $v^{*}$ and $v_{*}$ are upper and lower semicontinuous on $\bar{\Omega}$, respectively, with values in $\mathbb{R} \cup\{ \pm \infty\}$ and $v * \leq v \leq v^{*}$ in $\bar{\Omega}$. By $\operatorname{USC}(\Omega)$ and $\operatorname{LSC}(\Omega)$ we denote the set of all upper and lower semi-continuous functions on $\Omega$, respectively. It can be easily seen that if $v$ is upper or lower semi-continuous at $x \in \Omega$, then $v(x)=v^{*}(x)$ or $v(x)=v_{*}(x)$, respectively.

For an upper or lower semi-continuous function $v: \bar{\Omega} \rightarrow \mathbb{R}$, its super-jet $J_{\Omega}^{2,+} v$ or sub-jet $J_{\Omega}^{2,-} v$ of second order at $x$ on $\Omega$ is defined by

$$
J_{\Omega}^{2,+} v(x)=\left\{(p, X) \in \mathbb{R}^{n} \times \mathbf{S}(n) \mid v(x+h) \leq v(x)+\langle p, h\rangle+\frac{1}{2}\langle X h, h\rangle+o\left(|h|^{2}\right)(h \rightarrow 0)\right\}
$$

and

$J_{\Omega}^{2,-} v(x)=\left\{(p, X) \in \mathbb{R}^{n} \times \mathbf{S}(n) \mid v(x+h) \geq v(x)+\langle p, h\rangle+\frac{1}{2}\langle X h, h\rangle+o\left(|h|^{2}\right)(h \rightarrow 0)\right\}$,

respectively. The "closure" of $J_{\Omega}^{2,+}$ is defined by

$$
\bar{J}_{\Omega}^{2,+} v(x)=\left\{\begin{array}{l|l}
(p, X) \in \mathbb{R}^{n} \times \mathbf{S}(n) & \begin{array}{l}
\exists\left(x^{k}, p^{k}, X^{k}\right) \in \Omega \times \mathbb{R}^{n} \times \mathbf{S}(n) \\
\text { such that }\left(p^{k}, X^{k}\right) \in J^{2++} v(x) \text { and } \\
\left(x^{k}, v\left(x^{k}\right), p^{k}, X^{k}\right) \rightarrow(x, v(x), p, X)(k \rightarrow \infty)
\end{array}
\end{array}\right\}
$$

Similarly we can define the closure $\bar{J}_{\Omega}^{2,-}$ of $J_{\Omega^{2}}^{2,-}$

We consider the Dirichlet problem for fully nonlinear second order partial differential equations

$$
\left.\begin{array}{rl}
f\left(x, u(x), D u, D^{2} u\right)=0 & \text { in } \Omega \\
u(x)=0 & \text { on } \partial \Omega
\end{array}\right\}
$$

where $u: \Omega \rightarrow \mathbb{R}$ is the unknown function, $f: \Omega \times \mathbb{R} \times \mathbb{R}^{n} \times \mathbf{S}(n) \rightarrow \mathbb{R}$ is a given function which is locally bounded, the others are the same as mentioned in Section 1.

The function $f$ is said to be proper if

$$
f^{*}(x, r, p, X) \leq f_{*}(x, s, p, Y) \quad \text { whenever } r \leq s \text { and } Y \leq X
$$

where $r, s \in \mathbb{R}, x, p \in \mathbb{R}^{n}, X, Y \in \mathbf{S}(n)$, and $\mathbf{S}(\ddot{n})$ is equipped with its usual order (see $[3])$. 
Definition 2.1. Suppose that $f$ is proper. A locally bounded function $u$ is called a viscosity sub. or super-solution of $f=0$ in $\Omega$ if for all $x \in \Omega$

$$
f_{*}\left(x, u^{*}(x), p, X\right) \leq 0 \quad \text { for all }(p, X) \in J_{\Omega}^{2,+} u^{*}(x)
$$

or

$$
f^{*}\left(x, u_{*}(x), q, Y\right) \geq 0 \quad \text { for all }(q, Y) \in J_{\Omega}^{2,-} u_{*}(x)
$$

respectively. A locally bounded function $u$ is called a viscosity solution of $f=0$ in $\Omega$ if it is both a viscosity sub- and super-solution of $f=0$ in $\Omega$.

Proposition 2.2. Let $\Omega \subset \mathbb{R}^{n}$ be locally compact, $v \in \operatorname{USC}(\Omega)$ (or $\hat{v} \in \operatorname{LSC}(\Omega)$ ), $z \in \Omega$ and $(p, X) \in J_{\Omega}^{2,+} v(z)\left(\right.$ or $\left.(p, X) \in J_{\Omega}^{2,-} \hat{v}(z)\right)$. Suppose also that $u^{(k)}$ is a sequence of upper or lower semi-continuous functions on $\Omega$ such that:

(i) There exists $x^{(k)} \in \Omega$ such that $\left(x^{(k)}, u^{(k)}\left(x^{(k)}\right)\right) \rightarrow(z, v(z))($ or $(z, \hat{v}(z)))$.

(ii) If $z^{(k)} \in \Omega$ and $z^{(k)} \rightarrow x \in \Omega$, then

$$
\underset{k \rightarrow \infty}{\limsup } u^{(k)}\left(z^{(k)}\right) \leq v(x) \quad\left(\text { or } \liminf _{k \rightarrow \infty} u^{(k)}\left(z^{(k)}\right) \geq \hat{v}(x)\right)
$$

Then there exists

$$
\hat{x}^{(k)} \in \Omega \quad \text { and }\left(p^{(k)}, X^{(k)}\right) \in J_{\Omega}^{2,+} u^{(k)}\left(\hat{x}^{(k)}\right) \quad\left(\text { or }\left(p^{(k)}, X^{(k)}\right) \in J_{\Omega}^{2,-} u^{(k)}\left(\hat{x}^{(k)}\right)\right)
$$

such that

$$
\left(\hat{x}^{(k)}, u^{(k)}\left(\hat{x}^{(k)}\right), p^{(k)}, X^{(k)}\right) \rightarrow(z ; v(z), p, X)(\operatorname{or}(z, \hat{v}(z), p, X)) .
$$

As applications of Proposition 2.2, the following results can be casily proved.

Proposition 2.3. Suppose that $f$ is proper. A locally bounded function $u$ is $a$ viscosity sub-or super-solution of $f=0$ in $\Omega$ if and only if for all $x \in \Omega$

$$
f_{*}\left(x, u^{*}(x), p, X\right) \leq 0 \quad \text { for all }(p, X) \in \bar{J}_{\Omega}^{2,+} u^{*}(x)
$$

or

$$
f^{*}\left(x, u_{*}(x), q, Y\right) \geq 0 \quad \text { for all }(q, Y) \in \bar{J}_{\Omega}^{2,-} u_{*}(x) .
$$

Lemma 2.4. Suppose that $\Omega \subset \mathbb{R}^{n}$ is locally compact and $f \in \operatorname{LSC}\left(\Omega \times \mathbb{R} \times \mathbb{R}^{n} \times\right.$ $\mathrm{S}(n))$. Let

$$
\begin{gathered}
S=\{u \mid u \text { is a viscosity sub-solution of } f=0\} \\
\bar{u}=\sup \left\{u^{*} \mid u \in S\right\}
\end{gathered}
$$

and assume that $\bar{u}(x)<\infty$ for $x \in \Omega$. Then $\bar{u}$ is a viscosity sub-solution of $f=0$ in $\Omega$.

Lemma 2.5. Suppose that $\Omega \subset \mathbb{R}^{n}$ is locally compact and $f \in \operatorname{USC}\left(\Omega \times \mathbb{R} \times \mathbb{R}^{n} \times\right.$ $\mathrm{S}(n))$. Let

$$
\begin{gathered}
P=\{u \mid u \text { is a viscocity super-solution of } f=0\} \\
\bar{v}=\inf \left\{u_{*} \mid u \in P\right\}
\end{gathered}
$$


and assume that $-\bar{v}(x)<\infty$ for $x \in \Omega$. Then $\bar{v}$ is a viscosity super-solution of $f=0$ in $\Omega$.

Lemma 2.6. Let $\Omega \subset \mathbb{R}^{n}$ be open and $u$ be a viscosity sub-'(or super-solution) of $f=0$ in $\Omega$. If $u$ fails to be a super-solution (or sub-solution) at some point $\hat{x}$, then for any small $r>0$ there is another viscosity sub-solution (or super-solution) $w$ of $f=0$ in $\Omega$ satisfying

$$
\left.\begin{array}{l}
w(x) \geq u(x) \quad(x \in \Omega), \quad \sup _{\Omega}(w-u)>0 \\
\left(\text { or } w(x) \leq u(x) \quad(x \in \Omega), \quad \sup _{\Omega}(w-u)<0\right) \\
w(x)=u(x) \quad(x \in \Omega,|x-\hat{x}| \geq r)
\end{array}\right\} .
$$

In fact, we can follow the ideas in $[3,9]$ to prove Proposition 2.3 and Lemma 2.4 directly. The proof of Lemma 2.6 is similar to those of corresponding results in [3], and Lemma 2.5 may be easily proved if we choose $v=\hat{v}$ in conditions (i) and (ii) of Proposition 2.2.

Using the lemmas above, it can be proved that there exists a viscosity solution to problem (2.1).

Theorem 2.7. Let comparison hold for problem (2.1), i.e. if $U$ is a sub-and $V$ a super-solution of that problem, then $U^{*}(x) \leq V_{*}(x)$ for $x \in \bar{\Omega}$. Suppose that there exists a sub-solution $U$ and a super-solution $V$ of problem (2.1) as well satisfying the boundary condition $U_{*}(x)=V^{*}(x)=0$ for $x \in \partial \Omega$. Then $\bar{u} \equiv \bar{v} \in C(\bar{\Omega})$, as defined by $(2.2)$ and (2.3), is a unique viscosity solution of problem (2.1).

Proposition 2.8 (Comparison). Let $\Omega$ be a bounded open subset of $\mathbb{R}^{n}, f$ be proper, locally bounded and satisfying the following conditions:

(i) There exists $\gamma>0$ such that

$$
\gamma(r-s) \leq f_{*}(x, r, p, X)-f^{*}(x, s, p, X)
$$

for $r \geq s$ and $(x, p, X) \in \bar{\Omega} \times \mathbb{R}^{n} \times \mathbf{S}(n)$.

(ii) There exists a continuous function $\omega:(0, \infty) \rightarrow[0, \infty)$ that satisfies $\omega\left(0^{+}\right)=0$ such that

$$
f^{*}(y, r, \alpha(x-y), Y)-f_{*}(x, r, \alpha(x-y), X) \leq \omega\left(\alpha|x-y|^{2}+|x-y|\right)
$$

whenever $x, y \in \Omega, r \in \mathbb{R}$ and $X, Y \in \mathbf{S}(n)$ which satisfy

$$
-3 \alpha\left(\begin{array}{cc}
I & 0 \\
0 & I
\end{array}\right) \leq\left(\begin{array}{cc}
X & 0 \\
0 & -Y
\end{array}\right) \leq 3 \alpha\left(\begin{array}{rr}
I & -I \\
-I & I
\end{array}\right) .
$$

Let $u$ and $v$ be viscosity sub- and super-solutions of $f=0$ in $\Omega$, respectively, and $u^{*} \leq v$ * on $\partial \Omega$. Then $u^{*} \leq v$, on $\bar{\Omega}$.

Lemma 2.9 (see [3: Theorem 3.1]). Let $\Omega$ be a subset of $\mathbb{R}^{n}, u \in \operatorname{USC}(\Omega)$ and $v \in \operatorname{LSC}(\Omega)$, and

$$
M_{\alpha}=\sup _{\Omega}\left(u(x)-v(y)-\frac{\alpha}{2}|x-y|^{2}\right) \quad(\alpha>0) .
$$


Let $M_{\alpha}<\infty$ for large $\alpha$ and $\left(x_{\alpha}, y_{\alpha}\right)$ be such that

$$
\lim _{\alpha \rightarrow \infty}\left(M_{\alpha}-\left(u\left(x_{\alpha}\right)-v\left(y_{\alpha}\right)-\frac{\alpha}{2}\left|x_{\alpha}-y_{\alpha}\right|^{2}\right)\right)=0
$$

Then:

(i) $\lim _{\alpha \rightarrow \infty} \alpha\left|x_{\alpha}-y_{\alpha}\right|^{2}=0$.

(ii) $\lim _{\alpha \rightarrow \infty} M_{\alpha}=u(\hat{x})-v(\hat{x})=\sup _{\Omega}(u(x)-v(x))$ whenever $\hat{x} \in \Omega$ is a limit point of $x_{\alpha}$ as $\alpha \rightarrow \infty$.

Lemma 2.10 (see [3]). Let $\Omega$ be a locally compact subset of $\mathbb{R}^{n}, u \in \operatorname{USC}(\Omega)$ and $v \in \operatorname{LSC}(\Omega)$. Suppose that $(\hat{x}, \hat{y})$ is a local maximum of the function

$$
u(x)-v(y)-\frac{\alpha}{2}|x-y|^{2}
$$

relative to $\Omega$. Then for each $\varepsilon>0$ there exist $X, Y \in \mathbf{S}(n)$ satisfing (2.4) and

$$
\left.\begin{array}{c}
(\alpha(\hat{x}-\hat{y}), X) \in \bar{J}_{\Omega}^{2,+} u(\hat{x}) \\
(\alpha(\hat{x}-\hat{y}), Y) \in \bar{J}_{\Omega}^{2,-} v(\hat{y})
\end{array}\right\} .
$$

\section{Viscosity solutions of quasi-increasing systems}

We denote

$$
\left.\left.\begin{array}{l}
u=\left(u_{1}, u_{2}\right) \\
v=\left(v_{1}, v_{2}\right)
\end{array}\right\} \quad \text { and } \quad \begin{array}{l}
U=\left(U_{1}, U_{2}\right) \\
V=\left(V_{1}, V_{2}\right)
\end{array}\right\}
$$

where $u_{i}, v_{i} \quad(i=1,2)$ are functions from $\Omega$ into $\mathbb{R}$. The notation $u \leq v$ means $u_{i} \leq v_{i}(i=1,2)$. Also, denote

$$
\begin{aligned}
& u^{*}=\left(u_{1}^{*}, u_{2}^{*}\right) \\
& u_{*}=\left(u_{1 *}, u_{2 *}\right) .
\end{aligned}
$$

The pair $\left(F_{1}, F_{2}\right)$ is said to be proper if both

$$
\begin{aligned}
& F_{1}\left(x ;, u_{2} ; u_{1}, p_{1}, X_{1}\right) \\
& F_{2}\left(x ; u_{1} ; u_{2}, p_{2}, X_{2}\right)
\end{aligned}
$$

are proper with respect to the principal argument.

Definition 3.1. Let

$$
\left(F_{1}\left(x ;, u_{2} ; u_{1}, p_{1}, X_{1}\right), F_{2}\left(x ; u_{1} ; u_{2}, p_{2}, X_{2}\right)\right)
$$

be proper on $\Omega \times \mathbb{R}^{2} \times \mathbb{R}^{n} \times \mathbf{S}(n)$ and quasi-increasing. We call the pair $(\dot{U}, V)$ a couple of viscosity sub-and super-solutions of the Dirichlet problem (1.1) if $U$ and $V$ are locally bounded in $\Omega$ and such that

$$
\begin{aligned}
F_{i *}\left(x ; V_{j *} ; U_{i}^{*}, p_{i}, X_{i}\right) \leq 0 & & \left(\left(p_{i}, X_{i}\right) \in J_{\Omega}^{2,+} U_{i}^{*}(x)\right) \\
F_{i}^{*}\left(x ; U_{j}^{*} ; V_{i *}, q_{i}, Y_{i}\right) \geq 0 & & \left(\left(q_{1}, Y_{1}\right) \in J_{\Omega}^{2,-} V_{1 *}(x)\right)
\end{aligned}
$$


for all $x \in \Omega$ and $i, j=1,2$ with $j \neq i$ and

$$
U_{i}^{*} \leq 0 \leq V_{i *}
$$

for all $x \in \partial \Omega$ and $i=1,2$. And $U$ and $V$ are called viscosity sub-solution and supersolution of problem (1.1), respectively.

Definition 3.2. Let

$$
\left(F_{1}\left(x ;, u_{2} ; u_{1}, p_{1}, X_{1}\right), F_{2}\left(x ; u_{1} ; u_{2}, p_{2}, X_{2}\right)\right)
$$

be proper on $\Omega \times \mathbb{R}^{2} \times \mathbb{R}^{n} \times \mathbf{S}(n)$ and quasi-increasing. The pair $(U, V$. $)$ is called a viscosity coupled solution of the Dirichlet problem $(1.1)$ if $(U, V)$ and $(V, U)$ are both couples of viscosity sub- and super-solutions of (1.1).

Definition 3.3. Let $F_{1}$ and $F_{2}$ be proper and quasi-increasing. The function $u$ is called a viscosity solution of problem $(1.1)$ if $(u, u)$ is a viscosity coupled solution of (1.1).

We define

$$
\begin{aligned}
& S=S_{1} \times S_{2}=\left\{\begin{array}{l|l}
U=\left(U_{1}, U_{2}\right) & \begin{array}{l}
\exists V \text { such that }(U, V) \text { is a couple of } \\
\text { viscosity sub- and super-solutions of (1.1) }
\end{array}
\end{array}\right\} \\
& P=P_{1} \times P_{2}=\left\{\begin{array}{l|l}
V=\left(V_{1}, V_{2}\right) & \begin{array}{l}
\exists U \text { such that }(U, V) \text { is a couple of } \\
\text { viscosity sub- and super-solutions of (1.1) }
\end{array}
\end{array}\right\}
\end{aligned}
$$

and

$$
\left.\left.\begin{array}{l}
\bar{u}=\left(\bar{u}_{1}, \bar{u}_{2}\right) \\
\bar{v}=\left(\bar{v}_{1}, \bar{v}_{2}\right)
\end{array}\right\} \quad \text { where } \begin{array}{l}
\bar{u}_{i}(x)=\sup \left\{U_{i}(x) \mid U \in S\right\} \\
\bar{v}_{i}(x)=\inf \left\{V_{i}(x) \mid V \in P\right\}
\end{array}\right\} \quad(i=1,2) .
$$

Lemma 3.4. Assume that $F_{1}$ and $F_{2}$ are proper and quasi-increasing, and the sets $S, P$ are non-empty. If

$$
\left.\begin{array}{c}
\bar{u}_{i}^{*}(x)<+\infty \\
\bar{v}_{i *}(x)>-\infty
\end{array}\right\} \quad(x \in \bar{\Omega} ; i=1,2)
$$

then $\bar{u} \in S$ and $\bar{v} \in P$, i.e. $(\bar{u}, \bar{v})$ is a couple of viscosity sub- and super-solutions of problem (1.1).

Proof. Suppose that $z \in \Omega$ and $\left(p_{1}, X_{1}\right) \in J_{\Omega}^{2,+} \bar{u}_{1}^{*}(z)$. From the definition of $\bar{u}^{*}$, it is known that there is a sequence $\left\{u_{1}^{(k)}\right\} \subset S_{1}$ (we may suppose that all components of the sequence are upper semi-continuous) and a sequence of points $\left\{x^{(k)}\right\} \subset \Omega$ such that $\left(x^{(k)}, u_{1}^{(k)}\right) \rightarrow\left(z, \bar{u}_{i}^{*}\right)$. By virtue of upper semi-continuity of $\bar{u}_{i}^{*}(z)$, we have

$$
\limsup _{k \rightarrow \infty} u_{1}^{(k)}\left(y^{(k)}\right) \leq \bar{u}_{1}^{*}(z)
$$


for every sequence $\left(y^{(k)}, u_{1}^{(k)}\left(y^{(k)}\right)\right)$. From Proposition 2.2, there exist

$$
\hat{x}^{(k)} \in \Omega \quad \text { and } \quad\left(p_{1}^{(k)}, X_{1}^{(k)}\right) \in J_{\Omega}^{2,+} u_{1}^{(k)}\left(\hat{x}^{(k)}\right)
$$

such that

$$
\left(\hat{x}^{(k)}, u_{1}^{(k)}\left(\hat{x}^{(k)}\right), p_{1}^{(k)}, X_{1}^{(k)}\right) \rightarrow\left(z, \bar{u}_{1}^{*}(z), p_{1}, X_{1}\right) .
$$

From the definitions of $\bar{u}_{1}$ and $u_{1}^{(k)}$, there is $\bar{v}_{2}$ related $\bar{u}_{1}$ and $v_{2}^{(k)}$ related $u_{1}^{(k)}$. By virtue of lower semi-continuity of $\ddot{v}_{2 *}$, we know that

$$
\liminf _{k \rightarrow \infty} v_{2}^{(k)}\left(\hat{x}^{(k)}\right) \geq \bar{v}_{2 *}(z)
$$

Due to the quasi-monotonicity and semi-continuity of $F_{1}$,

$$
\begin{aligned}
F_{1 *}\left(z ; \bar{v}_{2 *}(z) ; \bar{u}_{1}^{*}(z), p_{1}, X_{1}\right) & \leq \liminf _{k \rightarrow \infty} F_{1 *}\left(\hat{x}^{(k)} ; v_{2}^{(k)}\left(\hat{x}^{(k)}\right) ; u_{1}^{(k)}\left(\hat{x}^{(k)}\right), p_{1}^{(k)} ; X_{1}^{(k)}\right) \\
& \leq 0
\end{aligned}
$$

Similarly, for $\left(q_{2}, Y_{2}\right) \in J_{\Omega}^{2,-} \bar{v}_{2 *}(z)$, there exists a sequence

$$
\left\{\left(y^{(j)}, v_{2}^{(j)}, q_{2}^{(j)}, Y_{2}^{(j)}\right)\right\} \subset \Omega \times P_{2} \times \mathbb{R}^{n} \times \mathbf{S}(n)
$$

such that

$$
\begin{aligned}
F_{2}^{*}\left(z ; \bar{u}_{1}^{*}(z) ; \bar{v}_{2 *}(z), q_{2}, Y_{2}\right) & \geq \limsup _{j \rightarrow \infty} F_{2}^{*}\left(y^{(j)} ; u_{1}^{(j)}\left(y^{(j)}\right) ; v_{2}^{(j)}\left(y^{(j)}\right), q_{2}^{(j)}, Y_{2}^{(j)}\right) \\
& \geq 0
\end{aligned}
$$

In the same way, we can also prove that, for all $z \in \Omega$,

$$
\begin{aligned}
F_{2 *}\left(z ; \bar{v}_{1 *}(z) ; \bar{u}_{2}^{*}(z), p_{2}, X_{2}\right) \leq 0 & & \left(\left(p_{2}, X_{2}\right) \in J_{\Omega}^{2,+} \bar{u}_{2}^{*}(z)\right) \\
F_{1}^{*}\left(z ; \bar{u}_{2}^{*}(z) ; \bar{v}_{1 *}(z), q_{1}, Y_{1}\right) \geq 0 & & \left(\left(q_{1}, Y_{1}\right) \in J_{\Omega}^{2,-} \bar{v}_{1 *}(z)\right) .
\end{aligned}
$$

The proof is completed

Lemma 3.5. Let $F_{1}$ and $F_{2}$ be proper and quasi-increasing. Assume that the sets $S, P$ are both non-empty. If $(u, v) \in S \times P$ while $(v, u) \notin S \times P$, then there is another $(U, V) \in S \times P$ such that $U \geq u, V \leq v$ and $(U, V) \neq(u, v)$.

Proof. If $(u, v) \in S \times P$ while $u_{1}$ is not a viscosity super-solution of $(3.1)$, then we know from.Lemma 2.6 [3: Lemma 4.4] that there exists another viscosity sub-solution of $(3.1) \hat{u}_{1}$ such that $\hat{u}_{1}(x) \geq u_{1}(x)$ and $\hat{u}_{1}(x) \neq u_{1}(x)$. Since $F_{2}$ is quasi-increasing,

$$
F_{2}^{*}\left(x ; \hat{u}_{1}^{*} ; v_{2 *}, q_{2}, Y_{2}\right) \geq F_{2}^{*}\left(x ; u_{1}^{*} ; v_{2}, q_{2}, Y_{2}\right) \geq 0
$$

for all $x \in \Omega$ and $\left(q_{2}, Y_{2}\right) \in J_{\Omega}^{2,-} v_{2 *}(x)$. Thus, there exist $U=\left(\hat{u}_{1}, u_{2}\right)$ and $V=v$ such that Lemma 3.5 holds. The other situations can be proved similarly 
Theorem 3.6. Suppose that $F_{1}$ and $F_{2}$ are both proper and quasi-increasing, and the sets $S$ and $P$ are both non-empty. If

$$
\left.\begin{array}{l}
\bar{u}_{i}^{*}(x)<\infty \\
\bar{v}_{i *}(x)>-\infty
\end{array}\right\} \quad(x \in \bar{\Omega} ; i=1,2)
$$

then $\bar{u} \in S$ and $\bar{v} \in P$, i.e. $(\bar{u}, \bar{v})$ is a viscosity coupled solution.

Proof. From Lemma 3.4 , we know that $(\bar{u}, \bar{v}) \in S \times P$. If $(\bar{v}, \bar{u}) \notin S \times P$, we know from Lemma 3.5 that there exists another $(U, V) \in S \times P$ such that $\bar{u}^{*} \leq U$ and $\bar{v}_{*} \geq V$, and $\left(\bar{u}^{*}, \bar{v}_{*}\right) \neq(U, V)$. This is a contradiction to the definition of $(\bar{u}, \bar{v})$. Hence, $(\bar{v}, \bar{u}) \in S \times P$

However, a viscosity coupled solution is not a real viscosity solution. Furthermore; we have the following theorem.

Theorem 3.7. Under the conditions of Theorem 3.6, if the comparison for viscosity sub-and super-solutions holds, i.e.

$$
u^{*}(x) \leq v_{*}(x) \quad(x \in \Omega) \quad \text { whenever }(u, v) \in S \times P
$$

then $\ddot{u} \equiv \bar{v} \in C(\Omega)$ is a unique viscosity solution of problem (1.1).

Proof. From the comparison we know that $S$ is bounded above and every $v \in P$ is an upper bound of $S$. Also, $P$ is bounded below and every $u \in S$ is a lower bound of $P$. From the definition of viscosity coupled solution, we know that $(\bar{u}, \bar{v}) \in S \times P$ and $(\bar{v}, \bar{u}) \in S \times P$. We have the inclusions

$$
\begin{array}{lll}
(\bar{u}, \bar{v}) \in S \times P & \Longrightarrow & \bar{u} \leq \bar{u}^{*} \leq \bar{v}_{*} \leq \bar{v} \\
(\bar{v}, \bar{u}) \in S \times P & \Longrightarrow & \bar{v} \leq \bar{v}^{*} \leq \bar{u}_{*} \leq \bar{u} .
\end{array}
$$

This implies that $\bar{u} \equiv \bar{v} \in C(\Omega)$. The uniqueness can be proved in the same way

\section{Viscosity solutions of mixed quasi-monotone systems}

First, we introduce the sub- and super-solutions of the system.

Definition 4.1. Let

$$
\left(F_{1}\left(x ;, u_{2} ; u_{1}, p_{1}, X_{1}\right), F_{2}\left(x ; u_{1} ; u_{2}, p_{2}, X_{2}\right)\right)
$$

be proper on $\Omega \times \mathbb{R}^{2} \times \mathbb{R}^{n} \times \mathbf{S}(n)$ and be mixed quasi-monotonic (we might always suppose as well that $F_{1}$ is quasi-decreasing and $F_{2}$ quasi-increasing when we talk about a mixed quasi-monotone system). Then

$$
\left.\begin{array}{l}
U(x)=\left(U_{1}(x), U_{2}(x)\right) \\
V(x)=\left(V_{1}(x), V_{2}(x)\right)
\end{array}\right\}
$$


are called a couple of viscosity sub-and super-solutions of the Dirichlet problem (1.1) if they are locally bounded in $\Omega$ and

$$
\begin{aligned}
F_{1 *}\left(x ; U_{2}^{*} ; U_{1}^{*}, p_{1}, X_{1}\right) \leq 0 & \forall\left(p_{1}, X_{1}\right) \in J_{\Omega}^{2,+} U_{1}^{*}(x) \\
F_{2 *}\left(x ; V_{1 *} ; U_{2}^{*}, p_{2}, X_{2}\right) \leq 0 & \forall\left(p_{2}, X_{2}\right) \in J_{\Omega}^{2,+} U_{2}^{*}(x) \\
F_{1}^{*}\left(x ; V_{2 *} ; V_{1 *}, q_{1}, Y_{1}\right) \geq 0 & \forall\left(q_{1}, Y_{1}\right) \in J_{\Omega}^{2,-} V_{1 *}(x) \\
F_{2}^{*}\left(x ; U_{1}^{*} ; V_{2 *}, q_{2}, Y_{2}\right) \geq 0 & \forall\left(q_{2}, y_{2}\right) \in J_{\Omega}^{2,-} V_{2 *}(x)
\end{aligned}
$$

for all $x \in \Omega$ and

$$
U_{i}^{*} \leq 0 \leq V_{i *} \quad(i=1,2)
$$

for all $x \in \partial \Omega$.

Similarly to Definitions 3.2 and 3.3 , we can define viscosity coupled solutions and viscosity solutions of mixed quasi-monotonic systems, as well as $S, P, \bar{u}_{i}$ and $\bar{v}_{i}$ (see (3.1) - (3.3)).

Lemma 4.2. Assume that $\left(F_{1}, F_{2}\right)$ is proper and mixed quasi-monotone, and the sets $S, P$ are non-empty. If

$$
\left.\begin{array}{c}
\bar{u}_{i}^{*}(x)<+\infty \\
\bar{v}_{i *}(x)>-\infty
\end{array}\right\} \quad(x \in \bar{\Omega} ; i=1,2)
$$

then $\bar{u} \in S$ and $\bar{v} \in P$, i.c. $(\bar{u}, \bar{v})$ is a couple of viscosity sub- and super-solutions of problem (1.1) as well.

Proof. Suppose that $z \in \Omega$ and $\left(q_{1}, Y_{1}\right) \in J_{\Omega}^{2,-} \bar{v}_{1 *}(z)$. From the definition of $\bar{v}_{*}$, we know that there exists a sequence $v_{1}^{(k)} \in P_{1}$ (we may as well suppose that they are lower semi-continuous) and points $x^{(k)} \in \Omega$ such that $\left(x^{(k)}, v_{l}^{(k)}\right) \rightarrow\left(z, \bar{v}_{1 *}\right)$. By virtue of lower semi-continuity of $\bar{v}_{1 *}(z)$, we see that

$$
\liminf _{k \rightarrow \infty} v_{1}^{(k)}\left(y^{(k)}\right) \geq \bar{v}_{1 *}(z)
$$

for every sequence $\left(y^{(k)}, v_{1}^{(k)}\left(y^{(k)}\right)\right)$. From Proposition 2.2, there exist

$$
\hat{x}^{(k)} \in \Omega \quad \text { and } \quad\left(q_{1}^{(k)}, Y_{1}^{(k)}\right) \in J_{\Omega}^{2,-} v_{1}^{(k)}\left(\hat{x}^{(k)}\right)
$$

such that

$$
\left(\hat{x}^{(k)}, v_{1}^{(k)}\left(\hat{x}^{(k)}\right), q_{1}^{(k)}, Y_{1}^{(k)}\right) \rightarrow\left(z, \bar{v}_{1 *}(z), q_{1}, Y_{1}\right)
$$

From the definition of $\vec{v}_{*}$, it follows that there exists a sequence of lower semi-continuous functions $v_{2}^{(k)} \in P_{2}$ such that $v_{2}^{(k)}\left(\hat{x}^{(k)}\right) \rightarrow \vec{v}_{2 *}(z)$. Thus, we know that

$$
\left(\hat{x}^{(k)}, v_{1}^{(k)}\left(\hat{x}^{(k)}\right), v_{2}^{(k)}\left(\hat{x}^{(k)}\right), q_{1}^{(k)}, Y_{1}^{(k)}\right) \rightarrow\left(z, \bar{v}_{1 *}(z), \bar{v}_{2 *}(z), q_{1}, Y_{1}\right)
$$


By using upper semi-continuity of $F_{1}^{*}$ and the fact that $\left(v_{1}^{(k)}, v_{2}^{(k)}\right) \in P$,

$$
\begin{aligned}
F_{1}^{*}\left(z ; \bar{v}_{2 *}(z) ; \bar{v}_{1 *}(z), q_{1}, Y_{1}\right) & \geq \limsup _{k \rightarrow \infty} F_{1}\left(\hat{x}^{(k)} ; v_{2}^{(k)}\left(\hat{x}^{(k)}\right) ; v_{1}^{(k)}\left(\hat{x}^{(k)}\right), q_{1}^{(k)}, Y_{1}^{(k)}\right) \\
& \geq 0
\end{aligned}
$$

Similarly, we can show that, for all $x \in \Omega$,

$$
\begin{aligned}
F_{1 *}\left(z ; \bar{u}_{2}^{*}(z) ; \bar{u}_{1}^{*}(z), p_{1}, X_{1}\right) \leq 0 & \forall\left(p_{1}, X_{1}\right) \in J_{\Omega}^{2,+} \bar{u}_{1}^{*}(z) \\
F_{2 *}\left(z ; \bar{v}_{1 *}(z) ; \bar{u}_{2}^{*}(z), p_{2}, X_{2}\right) \leq 0 & \forall\left(p_{2}, X_{2}\right) \in J_{\Omega}^{2,+} \bar{u}_{2}^{*}(z) \\
F_{2}^{*}\left(z ; \bar{u}_{1}^{*}(z) ; \bar{v}_{2 *}(z), q_{2}, Y_{2}\right) \geq 0 & \forall\left(q_{2}, Y_{2}\right) \in J_{\Omega}^{2,-} \bar{v}_{2 *}(z) .
\end{aligned}
$$

Thus, we have proved that $(\bar{u}, \bar{v}) \in S \times P$

In the same way to prove Lemma 3.5, we can prove the following lemma.

Lemma 4.3. Let $\left(F_{1}, F_{2}\right)$ be proper and mixed quasi-monotone, and the sets $S, P$ be both non-empty. If $(u, v) \in S \times P$ while $(v, u) \notin S \times P$, then there is another $(U, V) \in S \times P$ such that $U \geq u, V \leq v$ and $(U, V) \neq(u, v)$.

As in the proof of Theorem 3.6, we can use Lemmas 4.2 and 4.3 to prove the following

Theorem 4.4. Suppose that $\left(F_{1}, F_{2}\right)$ is proper and mixed quasi-monotone, and the sets $S$ and $P$ are both non-empty. If

$$
\left.\begin{array}{c}
\bar{u}_{i}^{*}(x)<\infty \\
\bar{v}_{i *}(x)>-\infty
\end{array}\right\} \quad(x \in \bar{\Omega} ; i=1,2)
$$

then $(\bar{u}, \bar{v})$ is a viscosity coupled solution of problem (1.1).

Corresponding Theorem 3.7, we have

Theorem 4.5. Under the conditions of Theorem 4.4, if the comparison for viscosity sub-and super-solutions holds, then $\bar{u} \equiv \bar{v} \in C(\Omega)$ is the unique viscosity solution of problem (1.1).

Remark. As for quasi-decreasing system, the same results can be obtained by use of our method. In addition, using our method, it can be shown without the comparison that problem (1.1) has two viscosity solutions $\bar{u}$ and $\bar{v}$, which are not necessarily identical and continuous. With the comparison, it can be proved that problem (1.1) has a unique continuous viscosity solution. 


\section{Comparison}

First, we consider the following conditions (A1) - (A4).

(A1) There exist $\gamma_{i}>0(i=1,2)$ such that

$$
\gamma_{i}\left(r_{i}-s_{i}\right) \leq F_{i *}\left(x ; u_{j} ; r_{i}, p, X\right)-F_{i}^{*}\left(x ; u_{j} ; s_{i}, p, X\right)
$$

or

$$
\gamma_{i}\left(r_{i}-s_{i}\right) \leq F_{i *}\left(x ; u_{j} ; r_{i}, p, X\right)-F_{i *}\left(x ; u_{j} ; s_{i}, p, X\right)
$$

or

$$
\gamma_{i}\left(r_{i}-s_{i}\right) \leq F_{i}^{*}\left(x ; u_{j} ; r_{i}, p, X\right)-F_{i}^{*}\left(x ; u_{j} ; s_{i}, p, X\right)
$$

for all $r_{i} \geq s_{i},\left(x ; u_{j} ; p, X\right) \in \bar{\Omega} \times \mathbb{R} \times \mathbb{R}^{n} \times \mathbf{S}(n)$ and $i, j=1,2$ with $j \neq i$.

(A2) There are modular functions $\omega_{i}:[0, \infty] \rightarrow[0, \infty](i=1,2)$ that satisfy $\omega_{i}\left(0^{+}\right)=0$ such that

$$
F_{i}^{*}\left(y ; u_{j} ; r_{i}, \alpha(x-y), Y\right)-F_{i *}\left(x ; u_{j} ; r_{i}, \alpha(x-y), X\right) \leq \omega_{i}\left(\alpha|x-y|^{2}+|x-y|\right)
$$

or

$$
F_{i *}\left(y ; u_{j} ; r_{i}, \alpha(x-y), Y\right)-F_{i *}\left(x ; u_{j} ; r_{i}, \alpha(x-y), X\right) \leq \omega_{i}\left(\alpha|x-y|^{2}+|x-y|\right)
$$

or

$$
F_{i}^{*}\left(y ; u_{j} ; r_{i}, \alpha(x-y), Y\right)-F_{i}^{*}\left(x ; u_{j} ; r_{i}, \alpha(x-y), X\right) \leq \omega_{i}\left(\alpha|x-y|^{2}+|x-y|\right)
$$

whenever $x, y \in \Omega, r_{i}, u_{j} \in \mathbb{R}$ and $X, Y \in \mathbf{S}(n)$ which satisfy (2.11) for $i, j=1,2$ with $j \neq i$.

(A3) There are constants $L_{\mathbf{3}} \geq 0 \quad(i=1,2)$ such that

$$
\left.F_{i}^{*}\left(x ; v_{j} ; r_{i}, p, X\right)-F_{i *}\left(x ; u_{j} ; r_{i}, p\right), X\right) \leq L_{i}\left(v_{j}-u_{j}\right)
$$

or

$$
F_{i *}\left(x ; v_{j} ; r_{i}, p, X\right)-F_{i *}\left(x ; u_{j} ; r_{i}, p, X\right) \leq L_{i}\left(v_{j}-u_{j}\right)
$$

or

$$
F_{i}^{*}\left(x ; v_{j} ; r_{i}, p, X\right)-F_{i}^{*}\left(x ; u_{j} ; r_{i}, p, X\right) \leq L_{i}\left(v_{j}-u_{j}\right)
$$

whenever $v_{j}, u_{j} \in \mathbb{R}$ with $v_{j} \geq u_{j}, r_{i} \in \mathbb{R}(j=1,2, j \neq i), x \in \Omega, p \in \mathbb{R}^{n}$ and $X \in \mathbf{S}(n)$. (A4) The constants $\gamma_{i}, L_{i}(i=1,2)$ in (A1) and (A3) satisfy $\frac{L_{2} L_{2}}{\gamma_{1} \gamma_{2}}<1$. 
Remark 1. It is clear that the first inequalities in (A1) - (A3) imply the other two since $F_{i}^{*} \geq F_{i *}$.

Remark 2. Similar conditions can be found in [3,9]. Actually, (A1) means that $F_{i *}$ or $F_{i}^{*} \quad(i=1,2)$ are strictly increasing with respect to the principal argument $u_{i}$, and (A2) implies that $F_{i *}$ or $F_{i}^{*}(i=1,2)$ are degenerated elliptic and uniformly continuous with respect to the rgument $x$ (see [3]). (A1) and (A2) are slightly stronger than weak properness. (A3) implies $F_{i *}$ or $F_{i}^{*}(i=1,2)$ are Lipschitz continuous with respect to the coupled argument $u_{j}(j \neq i)$ since they are quasi-increasing.

Remark-3. Saying that conditions (A1) - (A3) hold means one of the following combinations of three of nine inequalities in (A1) - (A3) holds:

$\begin{array}{lll}(A 1.1),(A 2.1),(A 3.1) & (A 1.1),(A 2.2),(A 3.2) & (A 1.1),(A 2.3),(A 3.3) \\ (A 1.2),(A 2.1),(A 3.2) & (A 1.2),(A 2.2),(A 3.1) & (A 1.2),(A 2.3),(A 3.1) \\ (A 1.3),(A 2.1),(A 3.2) & (A 1.3),(A 2.2),(A 3.1) & (A 1.3),(A 2.3),(A 3.1)\end{array}$

The following is the main result in this section.

Theorem 5.1. Let $F_{1}$ and $F_{2}$ be locally bounded, quasi-increasing and satisfy conditions (A1) - (A4). If $u$ and $v$ are a couple of viscosity sub-and super-solution of problem (1.1), then $u^{*} \leq v_{*}$ on $\bar{\Omega}$. that

Proof. If the comparison is not true, we may suppose without loss of generality

$$
\sup _{x \in \bar{\Omega}}\left\{u_{1}^{*}(x)-v_{1 *}(x)\right\}=\delta_{1}>0
$$

Let

$$
M(x, y)=u_{1}^{*}(x)-v_{1 *}(y)-\frac{\alpha}{2}|x-y|^{2} .
$$

For a fixed $\alpha$, we assume that $M(x, y)$ attains its local maximum point at $\left(x_{\alpha}, y_{\alpha}\right) \in$ $\Omega \times \Omega$ (denoted by $\left.M_{\alpha}=M\left(x_{\alpha}, y_{\alpha}\right)\right)$. From boundary condition, $\left(x_{\alpha}, y_{\alpha}\right) \notin \partial \Omega \times \partial \Omega$. Then

$$
u_{1}^{*}\left(x_{\alpha}\right)-v_{1 *}\left(y_{\alpha}\right) \geq M_{\alpha} \geq \delta_{1}>0 \text {. }
$$

From Lemma 2.10, there exist $p=\alpha\left(x_{\alpha}-y_{\alpha}\right)$ and $X, Y \in \mathbf{S}(n)$ such that

$$
\left.\begin{array}{l}
(p, X) \in \bar{J}_{\Omega}^{2,+} u_{1}^{*}\left(x_{\alpha}\right) \\
(p, Y) \in \bar{J}_{\Omega}^{2,-} v_{1 *}\left(y_{\alpha}\right)
\end{array}\right\}
$$

If conditions (A1.1), (A2.3), (A3.3) hold, then (A1.1) gives

$$
\begin{aligned}
\gamma_{1}\left(u_{1}^{*}(\right. & \left.\left.x_{\alpha}\right)-v_{1 *}\left(y_{\alpha}\right)\right) \\
\leq & F_{1 *}\left(x_{\alpha} ; v_{2 *}\left(x_{\alpha}\right) ; u_{1}^{*}\left(x_{\alpha}\right), p, X\right)-F_{1}^{*}\left(x_{\alpha} ; v_{2 *}\left(x_{\alpha}\right) ; v_{1 *}\left(y_{\alpha}\right), p, X\right) \\
\leq & F_{1 *}\left(x_{\alpha} ; v_{2 *}\left(x_{\alpha}\right) ; u_{1}^{*}\left(x_{\alpha}\right), p, X\right)-F_{1}^{*}\left(y_{\alpha} ; u_{2}^{*}\left(y_{\alpha}\right) ; v_{1 *}\left(y_{\alpha}\right), p, Y\right) \\
& +F_{1}^{*}\left(y_{\alpha} ; u_{2}^{*}\left(y_{\alpha}\right) ; v_{1 *}\left(y_{\alpha}\right), p, Y\right)-F_{1}^{*}\left(x_{\alpha} ; u_{2}^{*}\left(y_{\alpha}\right) ; v_{1 *}\left(y_{\alpha}\right), p, X\right) \\
& +F_{1}^{*}\left(x_{\alpha} ; u_{2}^{*}\left(y_{\alpha}\right) ; v_{1 *}\left(y_{\alpha}\right), p, X\right)-F_{1}^{*}\left(x_{\alpha} ; v_{2 *}\left(x_{\alpha}\right) ; v_{1 *}\left(y_{\alpha}\right), p, X\right) .
\end{aligned}
$$


Since $u, v$ is a couple of viscosity sub- and super-solution of problem (1.1),

$$
F_{1 *}\left(x_{\alpha} ; v_{2 *}\left(x_{\alpha}\right) ; u_{1}^{*}\left(x_{\alpha}\right), p ; X\right)-F_{1}^{*}\left(y_{\alpha} ; u_{2}^{*}\left(y_{\alpha}\right) ; v_{1 *}\left(y_{\alpha}\right), p, Y\right) \leq 0 .
$$

From (A2.3),

$$
\begin{gathered}
F_{1}^{*}\left(y_{\alpha} ; u_{2}^{*}\left(y_{\alpha}\right) ; v_{1 *}\left(y_{\alpha}\right), p, Y\right)-F_{1}^{*}\left(x_{\alpha} ; u_{2}^{*}\left(y_{\alpha}\right) ; v_{1 *}\left(y_{\alpha}\right), p, X\right) \\
\leq \omega_{1}\left(\alpha\left|x_{\alpha}-y_{\alpha}\right|^{2}+\left|x_{\alpha}-y_{\alpha}\right|\right) .
\end{gathered}
$$

Thus we have

$$
\begin{aligned}
0< & \gamma_{1} \delta_{1} \\
\leq & \gamma_{1}\left(u_{1}^{*}\left(x_{\alpha}\right)-v_{1 *}\left(y_{\alpha}\right)\right) \\
\leq & \omega_{1}\left(\alpha\left|x_{\alpha}-y_{\alpha}\right|^{2}+\left|x_{\alpha}-y_{\alpha}\right|\right) \\
& +F_{1}^{*}\left(x_{\alpha} ; u_{2}^{*}\left(y_{\alpha}\right) ; v_{1 *}\left(y_{\alpha}\right), p, X\right)-F_{1}^{*}\left(x_{\alpha} ; v_{2 *}\left(x_{\alpha}\right) ; v_{1 *}\left(y_{\alpha}\right), p, X\right) .
\end{aligned}
$$

Now we can assert that

$$
\sup _{x \in \bar{\Omega}}\left\{u_{2}^{*}(x)-v_{2 *}(x)\right\}=\delta_{2}>0 .
$$

Otherwise, from Lemma 2.9, we know $\alpha\left|x_{\alpha}-y_{\alpha}\right|^{2} \rightarrow 0$ as $\alpha \rightarrow \infty$. Observe from upper semi-continuity of $u_{2}^{*}(x)-v_{2 *}(y)$, we can deduce that, for $\alpha$ large enough, $u_{2}^{*}\left(y_{\alpha}\right) \leq$ $v_{2 *}\left(x_{\alpha}\right)$, i.c.

$$
F_{1}^{*}\left(x_{\alpha} ; u_{2}^{*}\left(y_{\alpha}\right) ; v_{1 *}\left(y_{\alpha}\right), p, X\right)-F_{1}^{*}\left(x_{\alpha} ; v_{2 *}\left(x_{\alpha}\right) ; v_{1 *}\left(y_{\alpha}\right), p, X\right) \leq 0
$$

since $F_{1}$ is quasi-increasing. Thus

$$
0<\gamma_{1} \delta_{1} \leq \gamma_{1}\left(u_{1}^{*}\left(x_{\alpha}\right)-v_{1 *}\left(y_{\alpha}\right)\right) \leq \omega_{1}\left(\alpha\left|x_{\alpha}-y_{\alpha}\right|^{2}+\left|x_{\alpha}-y_{\alpha}\right|\right) \rightarrow 0
$$

as $\alpha \rightarrow \infty$ which is a contradiction. So (5.5) holds, and $u_{2}^{*}\left(y_{\alpha}\right) \geq v_{2 *}\left(x_{\alpha}\right)$ for $\alpha$ large enough. Hence, we can use (A3.3) to obtain

$$
\begin{aligned}
\gamma_{1} \delta_{1} & \leq \gamma_{1}\left(u_{1}^{*}\left(x_{\alpha}\right)-v_{1 *}\left(y_{\alpha}\right)\right) \\
& \leq \omega_{1}\left(\alpha\left|x_{\alpha}-y_{\alpha}\right|^{2}+\left|x_{\alpha}-y_{\alpha}\right|\right)+L_{1}\left(u_{2}^{*}\left(y_{\alpha}\right)-v_{2 *}\left(x_{\alpha}\right)\right)
\end{aligned}
$$

Then we get

$$
\gamma_{1} \delta_{1} \leq L_{1} \delta_{2}
$$

as $\alpha \rightarrow \infty$. Similarly, starting from (5.5), we repeat the process above to get

$$
\gamma_{2} \delta_{2} \leq L_{2} \delta_{1} \text {. }
$$

Then we can deduce from (5.6), (5.7) and (A4) that

$$
0<\delta_{1} \leq \frac{L_{1}}{\gamma_{1}} \delta_{2} \leq \frac{L_{1} L_{2}}{\gamma_{1} \gamma_{2}} \delta_{1}<\delta_{1}
$$

which is a contradiction. So the comparison is true.

The proofs for the other combinations in conditions (A1) - (A3) (see Remark 3) will be similar, so we omitted them here 
Remark 4. In general, the conditions (A1) and (A2) are not necessary for the comparison to be hold. For instance, the problem $\Delta u=0$ in $\Omega, u=0$ on $\partial \Omega$, where although $u=\left(u_{1}, u_{2}\right)$ does not satisfy condition (A1), the comparison holds. However, (A1) and (A2) can be easily checked in general rather than to check the comparison directly.

Now we consider the comparison for mixed quasi-monotonic systems.

Theorem 5.2. Let $\left(F_{1}, F_{2}\right)$ be proper, mixed quasi-monotone and satisfying conditions (A1) - (A4). If $(U, V)$ is a couple of viscosity sub-and super-solution of problem (1.1), then $u^{*} \leq v_{*}$ on $\bar{\Omega}$.

Proof. If the comparison is not true, similar to the proof of Theorem 5.1, we may suppose that

$$
\sup _{x \in \bar{\Omega}}\left\{u_{1}^{*}(\bar{x})-v_{1 *}(x)\right\}=\delta_{1}>0
$$

Consider the function

$$
M(x, y)=u_{1}^{*}(x)-v_{1 *}(y)-\frac{\alpha}{2}|x-y|^{2}
$$

We assume that $\left(x_{\alpha}, y_{\alpha}\right)$ is the local maximum point of $M(x, y)$ for a fixed $\alpha>0$. It can be easily seen that

$$
u_{1}^{*}\left(x_{\alpha}\right)-v_{1 *}\left(y_{\alpha}\right) \geq M_{\alpha} \geq \delta_{1}>0
$$

where $M_{\alpha}=M\left(x_{\alpha}, y_{\alpha}\right)$.

Suppose we have conditions (A1.2), (A2.1) and (A3.2). Then, since $F_{1}$ is quasidecreasing and $F_{2}$ is quasi-increasing, we have from (A1.2)

$$
\begin{aligned}
& \gamma_{1}\left(u_{1}^{*}\left(x_{\alpha}\right)-v_{1 *}\left(y_{\alpha}\right)\right) \\
& \leq F_{1 *}\left(x_{\alpha} ; u_{2}^{*}\left(x_{\alpha}\right) ; u_{1}^{*}\left(x_{\alpha}\right), p, X\right)-F_{1 *}\left(x_{\alpha} ; u_{2}^{*}\left(x_{\alpha}\right) ; v_{1 *}\left(y_{\alpha}\right), p, X\right) \\
& \leq F_{1 *}\left(x_{\alpha} ; u_{2}^{*}\left(x_{\alpha}\right) ; u_{1}^{*}\left(x_{\alpha}\right), p, X\right)-F_{1}^{*}\left(y_{\alpha} ; v_{2 *}\left(y_{\alpha}\right) ; v_{1 *}\left(y_{\alpha}\right), p, Y\right) \\
&+F_{1}^{*}\left(y_{\alpha} ; v_{2 *}\left(y_{\alpha}\right) ; v_{1 *}\left(y_{\alpha}\right), p, Y\right)-F_{1 *}\left(x_{\alpha} ; v_{2 *}\left(y_{\alpha}\right) ; v_{1 *}\left(y_{\alpha}\right), p, X\right) \\
&+F_{1 *}\left(x_{\alpha} ; v_{2 *}\left(y_{\alpha}\right) ; v_{1 *}\left(y_{\alpha}\right), p, X\right)-F_{1 *}\left(x_{\alpha} ; u_{2}^{*}\left(x_{\alpha}\right) ; v_{1 *}\left(y_{\alpha}\right), p, X\right)
\end{aligned}
$$

where $(p, X) \in \bar{J}_{\Omega}^{2,+} u_{1}^{*}\left(x_{\alpha}\right)$ and $(p, Y) \in \bar{J}_{\Omega}^{2,-} v_{1 *}\left(y_{\alpha}\right)$. Since $u, v$ is a couple of viscosity sub- and super-solution of problem (1.1),

$$
F_{1 *}\left(x_{\alpha} ; u_{2}^{*}\left(x_{\alpha}\right) ; u_{1}^{*}\left(x_{\alpha}\right), p, X\right)-F_{1}^{*}\left(y_{\alpha} ; v_{2 *}\left(y_{\alpha}\right) ; v_{1 *}\left(y_{\alpha}\right), p, Y\right) \leq 0 .
$$

From (A2.1),

$$
\begin{gathered}
F_{1}^{*}\left(y_{\alpha} ; u_{2}^{*}\left(y_{\alpha}\right) ; v_{1 *}\left(y_{\alpha}\right), p, Y\right)-F_{1 *}\left(x_{\alpha} ; v_{2 *}\left(y_{\alpha}\right) ; v_{1 *}\left(y_{\alpha}\right), p, X\right) \\
\leq \omega_{1}\left(\alpha\left|x_{\alpha}-y_{\alpha}\right|^{2}+\left|x_{\alpha}-y_{\alpha}\right|\right)
\end{gathered}
$$


Thus, we have again that

$$
\begin{aligned}
0< & \gamma_{1} \delta_{1} \\
\leq & \gamma_{1}\left(u_{1}^{*}\left(x_{\alpha}\right)-v_{1 *}\left(y_{\alpha}\right)\right) \\
\leq & \omega_{1}\left(\alpha\left|x_{\alpha}-y_{\alpha}\right|^{2}+\left|x_{\alpha}-y_{\alpha}\right|\right) \\
& +F_{1 *}\left(x_{\alpha} ; v_{2}\left(y_{\alpha}\right) ; v_{1 *}\left(y_{\alpha}\right), p, X\right)-F_{1 *}\left(x_{\alpha} ; u_{2}^{*}\left(x_{\alpha}\right) ; v_{1 *}\left(y_{\alpha}\right), p, X\right) .
\end{aligned}
$$

Similar to the proof of Theorem 5.1 , we can assert that

$$
\sup _{x \in \bar{\Omega}}\left\{u_{2}^{*}(x)-v_{2 *}(x)\right\}=\delta_{2}>0
$$

and that $u_{2}^{*}\left(x_{\alpha}\right) \geq v_{2 *}\left(y_{\alpha}\right)$ for $\alpha$ large enough. Thus we have from (A3.2) that

$$
\begin{aligned}
\gamma_{1} \delta_{1} & \leq \gamma_{1}\left(u_{1}^{*}\left(x_{\alpha}\right)-v_{1 *}\left(y_{\alpha}\right)\right) \\
& \leq \omega_{1}\left(\alpha\left|x_{\alpha}-y_{\alpha}\right|^{2}+\left|x_{\alpha}-y_{\alpha}\right|\right)+L_{1}\left(u_{2}^{*}\left(x_{\alpha}\right)-v_{2 *}\left(y_{\alpha}\right)\right)
\end{aligned}
$$

Letting $\alpha \rightarrow \infty$, we obtain

$$
\gamma_{1} \delta_{1} \leq L_{1} \delta_{2}
$$

It is the same we can obtain as well

$$
\gamma_{2} \delta_{2} \leq L_{2} \delta_{1}
$$

Then, again from (5.12), (5.13) and condition (A4), we conclude the contrary inequality

$$
0<\delta_{1} \leq \frac{L_{1} L_{2}}{\gamma_{1} \gamma_{2}} \delta_{1}<\delta_{1}
$$

The proof is completed

Conditions (A1) - (A4) will also imply that the comparison holds in the case of quasi-decreasing systems. Thus combining the results in Sections 3 and 4 , we have the following obvious result.

Theorem 5.3. Let $\left(F_{1}, F_{2}\right)$ be proper and quasi-monotone (i.e. quasi-decreasing, increasing or mixed quasi-monotonic), and satisfy conditions (A1) - (A4). If (U,V) is a couple of viscosity sub-and super-solutions of problem (1.1), then this problem has a unique viscosity solution $u \in C(\Omega)$ satisfying $U^{*} \leq u \leq V$ * on $\bar{\Omega}$. 


\section{Viscosity solution for non-quasi-monotone systems}

In this section, we prove the existence, uniqueness and continuity of viscosity solution for non-quasi-monotonic systems. First, we introduce the concept of a so-called strong couple of viscosity sub- and super-solutions in the frame of locally bounded functions, which is similar to that introduced by [9] in the frame of semi-continuous functions. However, our concept here is more succinct and convenient.

Definition 6.1. Let $U(x)=\left(U_{1}(x), U_{2}(x)\right)$ and $V(x)=\left(V_{1}(x), V_{2}(x)\right)$ be locally bounded. Suppose that there exists a continuous function $w(x)=\left(w_{1}(x), w_{2}(x)\right)$ between $U(x)$ and $V(x)$, i.e. for all $x \in \Omega, U_{i}^{*}(x) \leq w_{i}(x) \leq V_{i *}(x)$ if $U_{i}^{*}(x) \leq V_{i *}(x)$ and $V_{i *}(x) \leq w_{i}(x) \leq U_{i}^{*}(x)$ if $V_{i *}(x) \leq U_{i}^{*}(x)$. Then $(U, V)$ is called a strong couple of viscosity sub-and super-solutions of problem (1.1) if for any continuous function $w(x)=\left(w_{1}(x), w_{2}(x)\right)$ which is between $U(x)$ and $V(x)$, we have

$$
F_{i *}\left(x ; w_{j} ; U_{i}^{*}, p_{i}, X_{i}\right) \leq 0 \leq F_{i}^{*}\left(x ; w_{j} ; V_{i *}, \dot{q}_{i}, Y_{i}\right)
$$

for all $x \in \Omega,\left(p_{i}, X_{i}\right) \in J_{\Omega}^{2,+} U_{i}^{*}(x)$ and $\left(q_{i}, Y_{i}\right) \in J_{\Omega}^{2,-} V_{i *}(x)$, and

$$
U_{i *}(x) \leq 0 \leq V_{i}^{*}(x)
$$

for all $x \in \partial \Omega$ and $i, j=1,2$ with $j \neq i$.

Lemma 6.2. Suppose conditions (A1) - (A3) hold. If $(U, V)$ is a strong couple of viscosity sub- and super-solutions of problem (1.1), then $U_{i}^{*}(x) \leq V_{i *}(x)$ for all $x \in \bar{\Omega}$ and $i=1,2$.

Proof. The proof is casy. In fact, if $w_{2}$ is a given function between $U_{2}$ and $V_{2}$, then $U_{1}$ and $V_{1}$ are the viscosity sub- and super-solutions, respectively, of the boundary value problem

$$
\left.\begin{array}{rl}
F_{1}\left(x ; w_{2} ; u, D u, D^{2} u\right) & =0 \\
u & =0 \quad(x \in \Omega) \\
& (x \in \partial \Omega)
\end{array}\right\} .
$$

Thus from Lemma 2.8 we have $U_{1}^{*}(x) \leq V_{1 *}(x) \quad(x \in \bar{\Omega})$. Similarly we may prove $U_{2}^{*}(x) \leq V_{2 *}(x) \quad(x \in \bar{\Omega})$

Theorem 6.3. Suppose conditions (A1) - (A4) hold. If there exists a strong couple of viscosity sub. and super-solutions of problem $(1.1)(U, V)$ which are bounded, then this problem has a unique viscosity solution $u \in C(\Omega)$ such that

$$
U_{i}^{*}(x) \leq u_{i}(x) \leq V_{i *}(x)
$$

for all $x \in \Omega$ and $i=1,2$.

Proof. For any $u \in C(\Omega)$ we define its norm as

$$
\|u\|=\sup _{\Omega}\left\{\left|u_{1}(x)\right|+\left|u_{2}(x)\right|\right\}
$$

and define

$$
B=\left\{u=\left(u_{1}, u_{2}\right) \in C(\Omega): U_{i}^{*}(x) \leq u_{i}(x) \leq V_{i *}(x) \quad(x \in \bar{\Omega} ; i=1,2)\right\} .
$$


From the assumption above, $B$ is a non-empty, bounded and close subset of $C(\Omega)$, and $\left\|V_{*}-U^{*}\right\|$ is bounded. For a given continuous function $\nu \in B$, consider the boundary value problems

$$
\left.\begin{array}{rl}
F_{i}\left(x ; \nu_{j} ; u_{i}, D u_{i}, D^{2} u_{i}\right) & =(x \in \Omega) \quad\left(\begin{array}{c}
i=1,2 \\
j \neq i
\end{array}\right) \\
u_{i} & =(x \in \partial \Omega)
\end{array}\right\} .
$$

Thus $U_{i}$ and $V_{i}$ are viscosity sub- and super-solutions of problem $(6.2)^{i}$, respectively. From Theorem 2.7, there exists a unique viscosity solution $u_{i}$ of $(6.2)^{i}$ which is continuous and satisfies $U_{i}^{*}(x) \leq u_{i}(x) \leq V_{i}(x)$ for all $x \in \bar{\Omega}$ and $i=1,2$ which implies $u \in B$. Thus we can define an operator $T$ on $B$ by $u=T \nu$. Obviously, a fixed point $u$ of $T$ on $B$ is a continuous viscosity solution of problem (1.1).

We can prove that $T$ has a unique fixed point by Banach fixed point theorem. Actually, we have the following lemma.

Lemma 6.4. Let $u$ be a unique fixed point of $T^{2}$. Then $u$ is a unique fixed point of $T$.

Proof. Let $u=T^{2} u=T(T u)$ and $v=T u$. Then $T^{2} v=T^{2}(T u)=T\left(T^{2} u\right)=$ $T u=v$, i.e. $v$ is a fixed point of $T^{2}$. From the uniqueness of the fixed point of $T^{2}$, we know that $u=v$ is a fixed point of $T$. If $w$ is another fixed point of $T$, then $T^{2} w=T(T w)=T w=w$, i.e. $w$ is also a fixed point of $T^{2}$, hence $w=u$

Thus, we only need to prove that $T^{2}$ is a deflatable. Let $\mu, \nu \in B$ and $u^{(k)}=T^{k} \mu$ as well as $v^{(k)}=T^{k} \nu(k=1,2)$. First, we suppose that

$$
\sup _{x \in \Omega}\left\{v_{1}^{(1)}(x)-u_{1}^{(1)}(x)\right\}>0 .
$$

Then from Lemma 2.9, there exists a point set $\left(x_{\alpha}, y_{\alpha}\right) \in \Omega \times \Omega$ such that $x_{\alpha}, y_{\alpha} \rightarrow \hat{x}$ as $\alpha \rightarrow \infty$ and

$$
v_{1}^{(1)}\left(x_{\alpha}\right)-u_{1}^{(1)}\left(y_{\alpha}\right) \geq v_{1}^{(1)}(\hat{x})-u_{1}^{(1)}(\hat{x})=\sup _{x \in \Omega}\left\{v_{1}^{(1)}(x)-u_{1}^{(1)}(x)\right\}>0 .
$$

From Lemma 2.10, there exist $p=\alpha\left(x_{\alpha}-y_{\alpha}\right)$ and $X_{1}^{v}, Y_{1}^{u}$ satisfying (2.4) such that

$$
\left.\begin{array}{l}
\left(p, X_{1}^{v}\right) \in J_{\Omega}^{2,+} v_{1}^{(1)}\left(x_{\alpha}\right) \\
\left(p, Y_{1}^{u}\right) \in J_{\Omega}^{2,-} u_{1}^{(1)}\left(y_{\alpha}\right)
\end{array}\right\} .
$$

If, without loss of generality, we have conditions (A1.3), (A2.3) and (A3.1), then inequality (A1.3) tells us that

$$
\begin{aligned}
& \gamma_{1}\left(v_{1}^{(1)}\left(x_{\alpha}\right)-u_{1}^{(1)}\left(y_{\alpha}\right)\right) \\
& \leq F_{1}^{*}\left(y_{\alpha} ; \mu_{2}\left(y_{\alpha}\right) ; v_{1}^{(1)}\left(x_{\alpha}\right), p, Y_{1}^{u}\right)-F_{1}^{*}\left(y_{\alpha} ; \mu_{2}\left(y_{\alpha}\right) ; u_{1}^{(1)}\left(y_{\alpha}\right), p, Y_{1}^{u}\right) \\
&= F_{1 *}\left(x_{\alpha} ; \nu_{2}\left(x_{\alpha}\right) ; v_{1}^{(1)}\left(x_{\alpha}\right), p, X_{1}^{v}\right)-F_{1}^{*}\left(y_{\alpha} ; \mu_{2}\left(y_{\alpha}\right) ; u_{1}^{(1)}\left(y_{\alpha}\right), p, Y_{1}^{u}\right) \\
& \quad+F_{1}^{*}\left(y_{\alpha} ; \mu_{2}\left(y_{\alpha}\right) ; v_{1}^{(1)}\left(x_{\alpha}\right), p, Y_{1}^{u}\right)-F_{1}^{*}\left(x_{\alpha} ; \mu_{2}\left(y_{\alpha}\right) ; v_{1}^{(1)}\left(x_{\alpha}\right), p, X_{1}^{v}\right) \\
& \quad+F_{1}^{*}\left(x_{\alpha} ; \mu_{2}\left(y_{\alpha}\right) ; v_{1}^{(1)}\left(x_{\alpha}\right), p, X_{1}^{v}\right)-F_{1 *}\left(x_{\alpha} ; \nu_{2}\left(x_{\alpha}\right) ; v_{1}^{(1)}\left(x_{\alpha}\right), p, X_{1}^{v}\right) .
\end{aligned}
$$


Next, from inequalities (A2.3) and (A3.1) we have

$$
v_{1}^{(1)}\left(x_{\alpha}\right)-u_{1}^{(1)}\left(y_{\alpha}\right) \leq \frac{1}{\gamma_{1}} \omega_{1}\left(\alpha\left|x_{\alpha}-y_{\alpha}\right|^{2}+\left|x_{\alpha}-y_{\alpha}\right|\right)+\frac{L_{1}}{\gamma_{1}}\left|\mu_{2}\left(y_{\alpha}\right)-\nu_{2}\left(x_{\alpha}\right)\right|
$$

Letting $\alpha \rightarrow \infty$ we get

$$
v_{1}^{(1)}(\hat{x})-u_{1}^{(1)}(\hat{x}) \leq \frac{L_{1}}{\gamma_{1}}\left|\mu_{2}(\hat{x})-\nu_{2}(\hat{x})\right|
$$

Also, starting from $\sup _{x \in \Omega}\left\{u_{1}^{(1)}(x)-v_{1}^{(1)}(x)\right\}>0$ we can get in the same way that

$$
u_{1}^{(1)}(\hat{x})-v_{1}^{(1)}(\hat{x}) \leq \frac{L_{1}}{\gamma_{1}}\left|\mu_{2}(\hat{x})-\nu_{2}(\hat{x})\right|
$$

Thus

$$
\left|v_{1}^{(1)}(\hat{x})-u_{1}^{(1)}(\hat{x})\right| \leq \frac{L_{1}}{\gamma_{1}}\left|\mu_{2}(\hat{x})-\nu_{2}(\hat{x})\right| .
$$

Similarly, we may deduce as well that

$$
\left|v_{2}^{(1)}(\hat{x})-u_{2}^{(1)}(\hat{x})\right| \leq \frac{L_{2}}{\gamma_{2}}\left|\mu_{1}(\hat{x})-\nu_{1}(\hat{x})\right|
$$

Replacing $\mu$ and $\nu$ by $u^{(1)}$ and $v^{(1)}$ in (6.3) and (6.4), respectively, we can obtain a similar estimate

$$
\left|v_{i}^{(2)}(\hat{x})-u_{i}^{(2)}(\hat{x})\right| \leq \frac{L_{i}}{\gamma_{i}}\left|v_{j}^{(1)}(\hat{x})-u_{j}^{(1)}(\hat{x})\right|
$$

for $i, j=1,2$ with $j \neq i$. Substituting (6.3) and (6.4) into (6.5), we have

$$
\left|v_{i}^{(2)}(\hat{x})-u_{i}^{(2)}(\hat{x})\right| \leq \frac{L_{1} L_{2}}{\gamma_{1} \gamma_{2}}\left|\mu_{i}(\hat{x})-\nu_{i}(\hat{x})\right| \quad(i=1,2) .
$$

Thus, we obtain

$$
\left\|v^{(2)}-u^{(2)}\right\|=\left\|T^{2} \nu-T^{2} \mu\right\| \leq \frac{L_{1} L_{2}}{\gamma_{1} \gamma_{2}}\|\nu-\mu\| .
$$

Due to condition (A4), $\frac{L_{1} L_{2}}{\gamma_{1} \gamma_{2}}<1$ which implies that $T^{2}$ is deflatable. According to the Banach fixed point theorem and Lemma 6.4, there is a unique fixed point $w$ of $T$, which is the unique viscosity solution of problem (1.1). Thus Theorem 6.3 is proved

Concerning the existence of a strong couple of viscosity sub- and super-solutions, we have the following

Theorem 6.5. Let inequalities (A1.2), (A1.3), (A3.2), (A3.3) and (A.4) hold. If $U$ and $V$ are standard viscosity sub-and super-solutions of problem (1.1), i.e.

$$
F_{i *}\left(x ; U_{j}^{*} ; U_{i}^{*}, p_{i}, X_{i}\right) \leq 0 \leq F_{i}^{*}\left(x ; V_{j *} ; V_{i *}, q_{i}, Y_{i}\right)
$$

for all $x \in \Omega$ and $\left(p_{i}, X_{i}\right) \in J_{\Omega}^{2,+} U_{i}(x),\left(q_{i}, Y_{i}\right) \in J_{\Omega}^{2,-} V_{i}(x)$, and

$$
U_{i}^{*}(x) \leq 0 \leq \dot{V}_{i *}(x)
$$

for all $x \in \partial \Omega$ and $i, j=1,2$ with $j \neq i$, then there exists a strong couple of viscosity sub-and super-solutions of problem (1.1). 
Proof. Let

$$
\begin{aligned}
& \hat{U}(x)=\left(\hat{U}_{1}(x), \hat{U}_{2}(x)\right)=\left(U_{1}^{*}(x)-b_{1}, U_{2}^{*}(x)-b_{2}\right) \\
& \hat{V}(x)=\left(\hat{V}_{1}(x), \hat{V}_{2}(x)\right)=\left(V_{1 *}(x)+c_{1}, V_{2 *}(x)+c_{2}\right)
\end{aligned}
$$

where $b_{1}, b_{2}, c_{1}$ and $c_{2}$ are non-negative constants, which will be determined later. Clearly, $\hat{U}_{i} \in \operatorname{USC}(\Omega)$ and $\hat{V}_{i} \in \operatorname{LSC}(\Omega)$, and

$$
\left.\begin{array}{l}
\hat{U}_{i}(x) \leq U_{i}^{*}(x) \\
\hat{V}_{i}(x) \geq V_{i *}(x)
\end{array}\right\} \quad(x \in \Omega ; i=1,2) .
$$

From inequalities (A1.2) and (A3.2), for all $(p, X) \in \mathbb{R}^{n} \times \mathbf{S}(n)$ and continuous function $\mu$ between $\hat{U}$ and $\hat{V}$, we have

$$
\begin{aligned}
F_{1 *}(x ; & \left.U_{2}^{*} ; U_{1}^{*}, p, X\right)-F_{1 *}\left(x ; \mu_{2} ; \hat{U}_{1}, p, X\right) \\
= & F_{1 *}\left(x ; U_{2}^{*} ; U_{1}^{*}, p, X\right)-F_{1 *}\left(x ; U_{2}^{*} ; \hat{U}_{1}, p, X\right) \\
& +F_{1 *}\left(x ; U_{2}^{*} ; \hat{U}_{1}, p, X\right)-F_{1 *}\left(x ; \mu_{2} ; \hat{U}_{1}, p, X\right) \\
& \geq \gamma_{1} b_{1}-L_{1}\left|\mu_{2}-U_{2}^{*}\right| .
\end{aligned}
$$

and

$$
\begin{aligned}
F_{2 *}(x ; & \left.U_{1}^{*} ; U_{2}^{*}, p, X\right)-F_{2 *}\left(x ; \mu_{1} ; \hat{U}_{2}, p, X\right) \\
= & F_{2 *}\left(x ; U_{1}^{*} ; U_{2}^{*}, p, X\right)-F_{2 *}\left(x ; U_{1}^{*} ; \hat{U}_{2}, p, X\right) \\
& \quad+F_{2 *}\left(x ; U_{1}^{*} ; \hat{U_{2}}, p, X\right)-F_{2 *}\left(x ; \mu_{1} ; \hat{U}_{2}, p, X\right) \\
\geq & \gamma_{2} b_{2}-L_{2}\left|\mu_{1}-U_{1}^{*}\right| .
\end{aligned}
$$

From inequalities (A1.3) and (A3.3), for all $(q, Y) \in \mathbb{R}^{n} \times \mathbf{S}(n)$ and continuous function $\nu$ between $\hat{U}$ and $\hat{V}$, we have

$$
\begin{aligned}
& F_{1}^{*}\left(x ; \nu_{2} ; \hat{V}_{1}, q, Y\right)-F_{1}^{*}\left(x ; V_{2 *} ; V_{1 *}, q, Y\right) \\
&= F_{1}^{*}\left(x ; \nu_{2} ; \hat{V}_{1}, q, Y\right)-F_{1}^{*}\left(x ; \nu_{2} ; V_{1 *}, q, Y\right) \\
& \quad+F_{1}^{*}\left(x ; \nu_{2} ; V_{1 *}, q, Y\right)-F_{1}^{*}\left(x ; V_{2 *} ; V_{1 *}, q, Y\right) \\
& \geq \gamma_{1} c_{1}-L_{1}\left|V_{2}-\nu_{2}\right|
\end{aligned}
$$

and

$$
\begin{aligned}
F_{2}^{*}(x ; & \left.\nu_{1} ; \hat{V}_{2}, q, Y\right)-F_{2}^{*}\left(x ; V_{1 *} ; V_{2 *}, q, Y\right) \\
= & F_{2}^{*}\left(x ; \nu_{1} ; \hat{V}_{2}, q, Y\right)-F_{2}^{*}\left(x ; \nu_{1} ; V_{2 *}, q, Y\right) \\
& +F_{2}^{*}\left(x ; \nu_{1} ; V_{2 *}, q, Y\right)-F_{2}^{*}\left(x ; V_{1 *} ; V_{2 *}, q, Y\right) \\
& \geq \gamma_{2} c_{2}-L_{2}\left|V_{1 *}-\nu_{1}\right| .
\end{aligned}
$$

In order to estimate $\left|\mu_{i}(x)-U_{i}^{*}(x)\right|$ and $\left|V_{i *}(x)-\nu_{i}(x)\right|(i=1,2)$ we let

$$
e_{i}=\sup _{x \in \Omega}\left|V_{i *}(x)-U_{i}^{*}(x)\right| \quad(i=1,2)
$$


Thus we know that

$$
\left|\mu_{i}(x)-U_{i}^{*}(x)\right| \leq\left\{\begin{array}{rl}
b_{i} & \text { if } \hat{\mathrm{U}}_{\mathrm{i}}(\mathrm{x}) \leq \mu_{\mathrm{i}}(\mathrm{x}) \leq \mathrm{U}_{\mathrm{i}}^{*}(\mathrm{x}) \\
c_{i}+e_{i} & \text { otherwise }
\end{array} \quad(x \in \Omega ; i=1,2) .\right.
$$

In the same way, we also have

$$
\left|V_{i *}(x)-\nu_{i}(x)\right| \leq\left\{\begin{aligned}
c_{i} & \text { if } V_{i *}(x) \leq \nu_{i}(x) \leq \hat{V}_{i}(x) \\
b_{i}+c_{i} & \text { otherwise }
\end{aligned} \quad(x \in \Omega ; i=1,2)\right.
$$

i.e. for $\hat{U}_{i}(x) \leq \mu_{i}(x) \leq U_{i}^{*}(x),(i=1,2)$,

$$
\begin{aligned}
& F_{1 *}\left(x ; U_{2}^{*} ; U_{1}^{*}, p, X\right)-F_{1 *}\left(x ; \mu_{2} ; \dot{\hat{U}}_{1}, p, X\right) \geq \gamma_{1} b_{1}-L_{1} b_{2} \\
& F_{2 *}\left(x ; U_{1}^{*} ; U_{2}^{*}, p, X\right)-F_{2 *}\left(x ; \mu_{1} ; \dot{U}_{2}, p, X\right) \geq \gamma_{2} b_{2}-L_{2} b_{1}
\end{aligned}
$$

or, otherwise,

$$
\begin{aligned}
& F_{1 *}\left(x ; U_{2}^{*} ; U_{1}^{*}, p, X\right)-F_{1 *}\left(x ; \mu_{2} ; \hat{U}_{1}, p, X\right) \geq \gamma_{1} b_{1}-L_{1}\left(c_{2}+e_{2}\right) \\
& F_{2 *}\left(x ; U_{1}^{*} ; U_{2}^{*}, p, X\right)-F_{2 *}\left(x ; \mu_{1} ; \hat{U}_{2}, p, X\right) \geq \gamma_{2} b_{2}-L_{2}\left(c_{1}+e_{1}\right) .
\end{aligned}
$$

Hence, on choosing

$$
\frac{L_{2}}{\gamma_{2}} b_{1} \leq b_{2} \leq \frac{\gamma_{1}}{L_{1}} b_{1}
$$

and

$$
\left.\begin{array}{l}
b_{1} \geq \frac{L_{1}}{\gamma_{1}}\left(c_{2}+e_{2}\right) \\
b_{2} \geq \frac{L_{2}}{\gamma_{2}}\left(c_{1}+e_{1}\right)
\end{array}\right\}
$$

we always have

$$
\begin{aligned}
& F_{1 *}\left(x ; U_{2}^{*} ; U_{1}^{*}, p, X\right)-F_{1 *}\left(x ; \mu_{2} ; \hat{U}_{1}, p, X\right) \geq 0 \\
& F_{2 *}\left(x ; U_{1}^{*} ; U_{2}^{*}, p, X\right)-F_{2 *}\left(x ; \mu_{1} ; \hat{U}_{2}, p, X\right) \geq 0
\end{aligned} .
$$

On the other hand, for $V_{i *}(x) \leq \nu_{i}(x) \leq \hat{V}_{i}(x) \quad(i=1,2)$ we have

$$
\begin{aligned}
& F_{1}^{*}\left(x ; \nu_{2} ; \hat{V}_{1}, q, Y\right)-F_{1}^{*}\left(x ; V_{2 *} ; V_{1 *}, q, Y\right) \geq \gamma_{1} c_{1}-L_{1} c_{2} \\
& F_{2}^{*}\left(x ; \nu_{1} ; \hat{V}_{2}, q, Y\right)-F_{2}^{*}\left(x ; V_{1 *} ; V_{2 *}, q, Y\right) \geq \gamma_{2} c_{2}-L_{2} c_{1}
\end{aligned}
$$

or, otherwise,

$$
\begin{aligned}
& F_{1}^{*}\left(x ; \nu_{2} ; \hat{V}_{1}, q, Y\right)-F_{1}^{*}\left(x ; V_{2 *} ; V_{1}, q, Y\right) \geq \gamma_{1} c_{1}-L_{1}\left(b_{2}+e_{2}\right) \\
& F_{2}^{*}\left(x ; \nu_{1} ; \hat{V}_{2}, q, Y\right)-F_{2}^{*}\left(x ; V_{1 *} ; V_{2 *}, q, Y\right) \geq \gamma_{2} c_{2}-L_{2}\left(b_{1}+e_{1}\right)
\end{aligned}
$$

Hence, if we choose

$$
\frac{L_{2}}{\gamma_{2}} c_{1} \leq c_{2} \leq \frac{\gamma_{1}}{L_{1}} c_{1}
$$


and

$$
\left.\begin{array}{l}
c_{1} \geq \frac{L_{1}}{\gamma_{1}}\left(b_{2}+e_{2}\right) \\
c_{2} \geq \frac{L_{2}}{\gamma_{2}}\left(b_{1}+e_{1}\right)
\end{array}\right\}
$$

then we have

$$
\begin{aligned}
& F_{1}^{*}\left(x ; \nu_{2} ; \hat{V}_{1}, q, Y\right)-F_{1}^{*}\left(x ; V_{2 *} ; V_{1 *}, q, Y\right) \geq 0 \\
& F_{2}^{*}\left(x ; \nu_{1} ; \hat{V}_{2}, q, Y\right)-F_{2}^{*}\left(x ; V_{1 *} ; V_{2 *}, q, Y\right) \geq 0
\end{aligned}
$$

Observe that

$$
\left.\begin{array}{l}
J_{\Omega}^{2, \pm} \hat{U}_{i}(x)=J_{\Omega}^{2, \pm} U_{i}^{*}(x) \\
J_{\Omega}^{2, \pm} \hat{V}_{i}(x)=J_{\Omega}^{2, \pm} V_{i *}(x)
\end{array}\right\} \quad(x \in \Omega ; i=1,2)
$$

and that $U$ and $V$ are standard viscosity sub- and super-solutions of problem (1.1). Thus for any continuous function $\mu=\left(\mu_{1}, \mu_{2}\right)$ which is between $\hat{U}$ and $\hat{V}$ we have from (6.9) and (6.12) that, for all $x \in \Omega$,

$$
\begin{aligned}
F_{1 *}\left(x ; \mu_{2} ; \hat{U}_{1}, p, X\right) \leq 0 & \left((p, X) \in J_{\Omega}^{2,+} \hat{U}_{1}(x)\right) \\
F_{2 *}\left(x ; \mu_{1} ; \hat{U}_{2}, p, X\right) \leq 0 & \left((p, X) \in J_{\Omega}^{2,+} \hat{U}_{2}(x)\right) \\
F_{1}^{*}\left(x ; \mu_{2} ; \hat{V}_{1}, q, Y\right) \geq 0 & \left((q, Y) \in J_{\Omega}^{2,-} \hat{V}_{1}(x)\right) \\
F_{2}^{*}\left(x ; \mu_{1} ; \hat{V}_{2}, q, Y\right) \geq 0 & \left((q, Y) \in J_{\Omega}^{2,-} \hat{V}_{2}(x)\right) .
\end{aligned}
$$

This implies that $\hat{U}, \hat{V}$ is a strong couple of viscosity sub- and super-solutions of problem (1.1).

Next, condition (A4) can ensure the existence of constants $b_{1}, b_{2}$ and $c_{1}, c_{2}$ such that inequalities $(6.7),(6.8)$ and $(6.10),(6.11)$ hold simultaneously, e.g. we can choose

$$
\begin{aligned}
& b_{1}=c_{1}=\frac{L_{1} L_{2} e_{1}+\gamma_{2} L_{1} e_{2}}{\gamma_{1} \gamma_{2}-L_{1} L_{2}} \\
& b_{2}=c_{2}=\frac{L_{1} L_{2} e_{2}+\gamma_{1} L_{2} e_{1}}{\gamma_{1} \gamma_{2}-L_{1} L_{2}} .
\end{aligned}
$$

The proof of Theorem 6.5 is complete

\section{Examples}

In this section, we present two examples.

Example 1. Let

$$
\left.\begin{array}{rl}
-\Delta u_{1}+u_{1}+a u_{2}=0 & \\
-\Delta u_{2}+u_{2}-1=0 & (x \in \Omega) \\
u_{1}=0 & \\
u_{2}=0 & (x \in \partial \Omega)
\end{array}\right\}
$$

System (7.1) is not quasi-decreasing ("quasi-monotone" in term of [9]) if $a>0$ (i.e. the condition of Theorems 3.3 in [9] is not satisfied). Indeed, in this case (7.1) is 
quasi-increasing. Furthermore, if $a>1$, the condition (A.1) of [9: Theorem 4.4] is not satisfied. So, we cannot prove existence of a viscosity solution by using the result of [9] directly. On the other hand, from standard elliptic partial differential equation theory we may easily prove that system (7.1) has a unique classical solution.

However, it is easy to see that the conditions in our Sections 3 and 5 are satisfied if we choose $U=(-2,0)$ and $V=(0,1)$ for $a>1$. Thus we can prove that system (7.1) has a unique viscosity solution $u=\left(u_{1}, u_{2}\right)$ by Theorem 5.3 here. In fact, $u$ is the unique classical solution as well, satisfying, by the classical maximum principle, $u_{2} \geq 0, u_{2} \not \equiv 0$ and $u_{1} \leq 0, u_{1} \not \equiv 0$ on $\bar{\Omega}$.

Example 2. Consider the weakly coupled system

$$
\begin{aligned}
G_{1}\left(x, D u_{1}, D^{2} u_{1}\right)+a u_{1}+b u_{2}=0 \\
G_{2}\left(x, D u_{2}, D^{2} u_{2}\right)+c u_{1}+d u_{2}=0 \\
u_{1}=0 \\
u_{2}=0
\end{aligned} \quad(x \in \Omega)
$$

System (7.2) is quasi-decreasing if $b \leq 0$ and $c \leq 0$. It is quasi-increasing if $b \geq 0$ and $c \geq 0$, and mixed quasi-monotonic if $b c \leq 0$. Clearly, for the later two cases. we cannot use directly the result of [9: Theorem 3.3]. Also in the case of $a<b$ and $d<c$ we cannot use the result of [9: Theorem 4.4]. If $a>0, d>0$ and $\frac{b c}{a d}<1$, system (7.2) satisfies conditions (A1) - (A4). Using our Theorem 5.3 (if $b \geq 0, c \geq 0$, or $b c \leq 0$ ) and combining with the viscosity sub- and super-solutions, we may obtain the existence of a viscosity solution.

Example 3 (switching games $[10,14,20,21]$ ). Consider the problem

$$
\begin{gathered}
\min \left\{\max \left\{G_{i}\left(x, u_{1}, D u_{1}, D^{2} u_{1}\right), M_{i}\left(x ; u_{1}, u_{2}\right)\right\}, N_{i}\left(x ; u_{1}, u_{2}\right)\right\}=0(x \in \Omega) \\
u_{i}=\phi_{i}(x)(x \in \partial \Omega ; i=1,2)
\end{gathered}
$$

where $G_{i}$ are proper and $\phi_{i}$ are continuous. Let $G_{i}$ satisfy conditions (A1) and (A2) with constants $\gamma_{i}, M_{i}$ and $N_{i}$ satisfy condition (A3) with constants $L_{i}$, and $\gamma_{i}$ and $L_{i}$ satisfy condition (A4). Then the existence and uniqueness of viscosity solutions for system (7.3) can be arrived at by our results, no matter whether (7.3) is quasi-monotone or not.

Acknowledgement. The authors are grateful to Prof. Qi Minyou (M. Y. Chi) for his encouragement. The paper was partially supported by the research group "Partial Differential Equations and Complex Analysis", University of Potsdam (Germany) during November 1999 while Chen Hua was a visiting professor at the Institute of Mathematics, University of Potsdam. He would like to thank the Institute for the invitation and hospitality. 


\section{References}

[1]. Crandall, M. G., Evans, L. C. and Lions, P. L.: Some properties of viscosity solutions of Hamilton-Jacobi equations. Trans. Amer. Math. Soc. 282 (1984), 487 - 502.

[2] Crandall, M. G. and Ishii, H.: The maximum principle for semi-continuous functions. Diff. Int. Eqs. 3 (1990), $1001-1014$.

[3] Crandall, M G., Ishii, H. and Lions, P. L.: User's guide to viscosity solutions of second order partial differential equations. Bull. Amer. Math. Soc. (new series) 27 (1992), 1 67.

[4] Crandall, M. G. and Lions, P. L.: Viscosity solutions of Hamilton-Jacobi equations. Trans. Amer. Math. Soc. 277 (1983), $1-42$.

[5] Engler, H. and Lenhart, S. M.: Viscosity solutions for weakly coupled systems of HamiltonJacobi equations. Proc. London Math. Soc. 63 (1991), 212 - 240.

[6] Guo Dajun and Lakshmikantham, V.: Coupled fixed point of nonlinear operators with applications. Nonlin. Anal.: Theo. Meth. Appl. 11 (1987), 623-632.

[7] Ishii, H.: Perron's method for Hamilton-Jacobi equations. Duke Math. J. 55 (1987), 369 -384 .

[8] Ishii, H.: On uniqueness and existence of viscosity solutions of fully nonlinear second order elliptic PDE's. Comm. Pure Appl. Math. 42 (1989), 15 - 45.

[9] Ishii, H. and Koike, S.: Viscosity solutions for monotone systerns of second-order elliptic $P D E$ 's. Comm. Part. Diff. Equ. 16 (1991), 1095 - 1128.

[10] Ishii, H. and Koike, S.: Viscosity solutions of a systems of nonlinear second-order elliptic $P D E$ 's arising in suitching games. Funkcial. Ekvac. 34 (1991), $143-155$.

[11] Jensen, R.: The maximum principle for viscosity solutions of fully nonlinear second order partial differential equations. Arch. Rat. Mech. Anal. 101 (1988), 1- 27.

[12] Koike, S.: Uniqueness of viscosity solutions for monotone systerns of fully nonlinear PDEs under Dirichlet condition. Nonlin. Anal.: Theo. Meth. Appl. 22 (1994), $519-532$.

[13] Lenhart, S. M.: Viscosity solutions for weakly coupled systems of first order PDEs. J. Math. Anal. Appl. 131 (1988), $180-193$.

[14] Lenhart, S. M. and Belbas, S. A.: A system of nonlinear partial differential equations arising in the optimal control of stochastic systems with switching costs. SIAM J. Appl. Math. 43 (1983), $465-475$.

[15] Li Zheng-yuan: Dominant equations of reaction-diffusion equations and applications (in Chinese). J. Peking Univ. (Nat. Sci. Edit.) 4 (1984), $13-26$.

[16] Liang Jin: Reaction-diffusion systems without monotone conditions. $\Lambda$ dv. Math. 14 (1985), $73-75$.

[17] Lions, P. I.: Optimal control of diffusion processes and Hamilton-Jacobi-Bellman equations. Part 2: Viscosity solutions and uniqueness. Comm. Part. Diff. Equ. 8 (1983), $1229-1276$.

[18] Liu Wei-an: Nonlocal problem of parabolic equations (in Chinese). J. Wuhan Univ. (Nat. Sci. Edit.) 42 (1996), $261-268$.

[19] Liu Wei-an: Viscosity Solutions for Fully Nonlinear Degenerated Elliptic and Parabolic Systems of Second Order PDEs (in Chinese). Thesis. Wuhan Univ. 1997.

[20] Liu Wei-an, Yang Ying and Chen Hua: Coupled fixed point of skew-increasing operators in Banach space. Preprint. 
[21] Yamada, N.: A system of elliptic variational inequalities associated with a stochastic switching game. Hiroshima Math. J. 13 (1983), $109-132$.

[22] Yamada, N.: Viscosity solutions for a system of inequalities with = bilateral obstacles. Funkcial Ekvac. 30 (1987), $417-425$.

[23] Yc Qi-xiao and Li Zheng-yuan: Introduction of Reaction-Diffusion Equations (in Chinese). Beijing: Science Press 1994.

Received 08.11.1999 